\title{
Solitary fibrous tumor of the inguinal region displaying heterogeneous echogenicity and its correlation with histopathologic findings
}

\begin{abstract}
Ai Kajita1, Yoshio Kawakami², Tomoko Miyake², Wakako Oda², Osamu Yamasaki', Shin Morizane ${ }^{1}$
\end{abstract}

${ }^{1}$ Department of Dermatology, Okayama University Graduate School of Medicine, Dentistry and Pharmaceutical Sciences, Okayama, Japan, ${ }^{2}$ Department of Pathology, Okayama City Hospital, Okayama, Japan

Corresponding author: Dr. Yoshio Kawakami, E-mail: kawayoshio@yahoo.co.jp

Sir,

Solitary fibrous tumor (SFT) — first documented by Klemperer and Rabin in 1931 as a primary neoplasm of the pleura [1]—represents a rare fibroblastic neoplasm composed of cellular areas and a less cellular zone of thick hyalinized collagen intermingled with numerous vessels. SFT is anatomically ubiquitous and classically benign, and malignant transformations have been reported [2]. The NAB2-STAT6 gene fusion, which is associated with nuclear relocation of STAT6 protein, has recently been identified as the defining driver mutation of SFT [3]. Herein, we report a case of SFT of the inguinal region and evaluate the correlation between ultrasonographic and histopathologic findings.

A 63-year-old male presented with a three-month history of a painless and mobile subcutaneous mass in the right inguinal region. Color Doppler revealed a well-circumscribed, ovoid, heterogeneous echoic mass $21 \times 31 \times 14 \mathrm{~mm}$ in size with peripheral color flow signals (Fig. la). Magnetic resonance imaging (MRI) showed low signal intensity on Tl-weighted image and high signal intensity on T2-weighted image. Computed tomography (CT) of the chest, abdomen, and pelvis showed no evidence of metastasis. The patient underwent excision of the lesion, which was located in the subcutaneous fat just below the superficial fascia (Fig. 1b). Histopathologic findings revealed an encapsulated tumor composed of cellular areas with patternless bland spindle cells without nuclear atypia and less cellular zones with abundant collagen fibers (Figs. lc - le). There were numerous vessels including a staghorn-like vascular network (Fig. ld) and clustering dilated vessels on the periphery (Fig. lf). Immunohistochemical staining showed cytoplasmic expression of CD34 (Fig. lg) and nuclear translocation of STAT6 protein (Fig. 1h), whereas expression of Bcl2 and CD99 was partial, and staining for AEl/AE3, desmin, S100 protein, c-KIT, factor VIII, and CD31 was negative (Fig. li). Based on these results, the patient was diagnosed with SFT. Since the surgical excision, there has been no recurrence for more than two years.

We have evaluated the correlation between ultrasonographic and histopathologic findings. In ultrasonography (USG), hypoechoic lesions matched the distribution of cellular areas in histology, whereas hyperechoic lesions matched the distribution of less cellular zones with abundant collagen fibers (Figs. la - le). Peripheral color flow signals in color Doppler ultrasonography corresponded to the clustering dilated vessels (Figs. la, lc, and lf).

The ultrasonographic findings of SFT have not been described due to its rarity and ubiquitous locations. Since the proportion of cellular areas and less cellular zones in SFT varies, the heterogenous pattern of SFT may vary from case to case. There have been several reports describing the ultrasonographic and color Doppler findings of SFT: solitary, homogenous hypoechoic lesions of the pleura in three cases [4]; an ovoid, hypoechoic lesion of the breast with peripheral and internal color flow signals [5]; and a mosaic echo

\footnotetext{
How to cite this article: Kajita A, Kawakami Y, Miyake T, Oda W, Yamasaki O, Morizane S. Solitary fibrous tumor of the inguinal region displaying heterogeneous echogenicity and its correlation with histopathologic findings. Our Dermatol Online. 2020;11(4):424-426.

Submission: 29.06.2020; Acceptance: 01.10.2020

DOI: 10.7241 /ourd.20204.28
} 


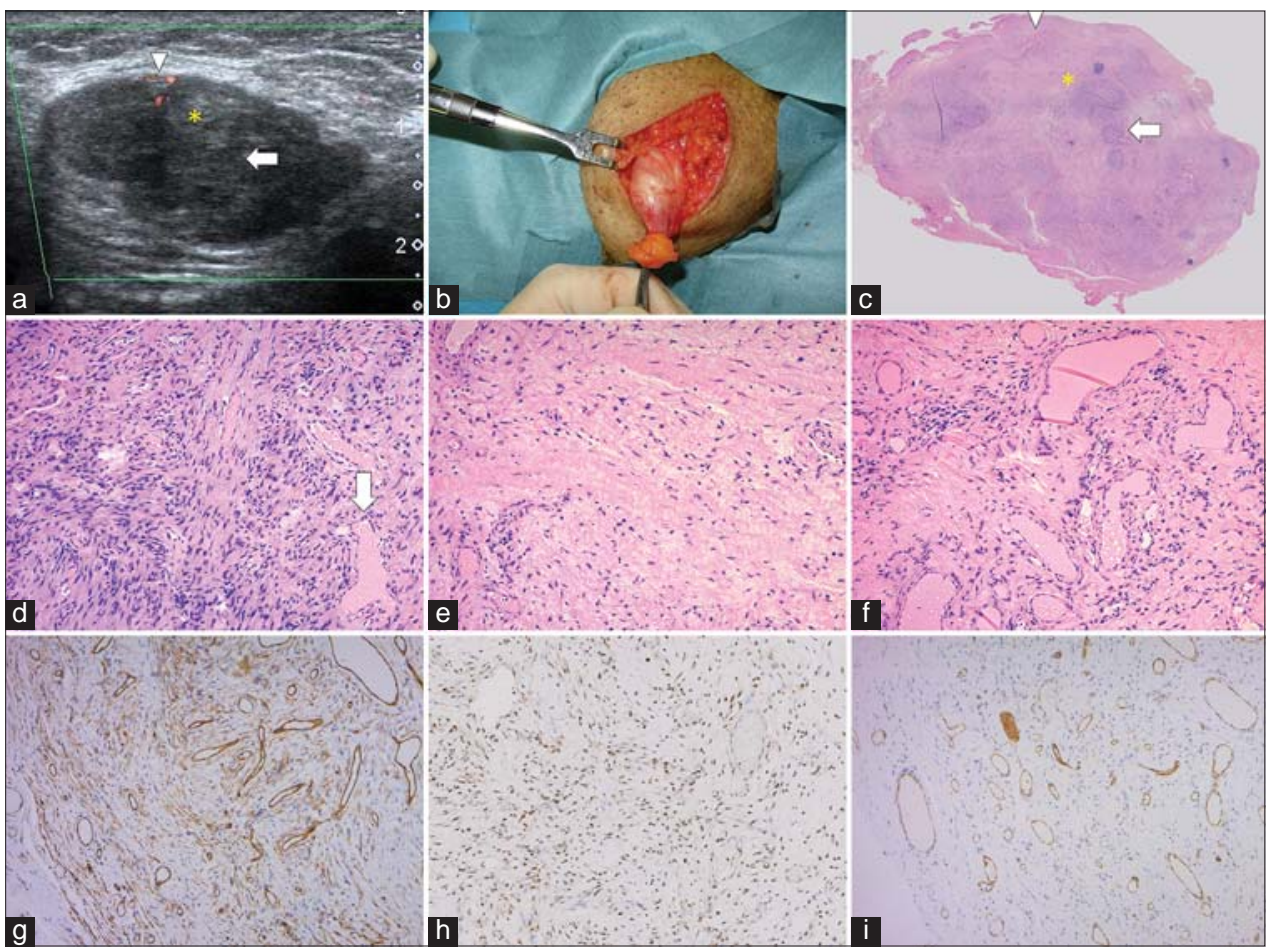

Figure 1: (a) Color Doppler USG showing an ovoid lesion with hypo- (arrow) and hyperechoic lesions (asterisk) and peripheral color flow signals (arrowhead).(b) Intraoperative findings showing an encapsulated subcutaneous tumor just below the superficial fascia. (c) An overview section displaying an encapsulated tumor composed of cellular areas (arrow) matching the distribution of hypoechoic lesions in USG and less cellular zones (asterisk) corresponding to the distribution of hyperechoic lesions in USG, as well as clustering dilated vessels on the periphery (arrowhead) matching the distribution of color signals on color Doppler (H\&E). (d) A high-power view of a cellular area (arrow in Fig. 1c) showing patternless spindle cells and numerous vessels, including staghorn-like vessels (arrow) (H\&E; original magnification: 100x). (e) A high-power view of a less cellular zone (asterisk in Fig. 1c) showing abundant collagen fibers (H\&E; original magnification: 100x). (f) A high-power view of the peripheral region (arrowhead in Fig. 1c) showing clustering dilated vessels (H\&E; original magnification: 100x). (g) Tumor cells showing positivity for CD34 (original magnification: 100x). (h) Tumor cells showing nuclear expression of STAT6 (1:200; sc-621; Santa Cruz) and vascular endothelial cells showing negative expression (original magnification: 100x). (i) Tumor cells showing negative expression of CD31, staining only vascular endothelial cells (original magnification: 100x).

pattern with rich blood flow signals in a subcutaneous tumor of the hip [6]. Two of them reported correlations between ultrasonographic and histopathologic or macroscopic findings. In both, the vasculature of the lesions in histologic or macroscopic findings matched the distribution of color flow signals on color Doppler $[5,6]$. Heterogeneous echoic masses with color flow signals in USG observed in our case have also been reported in other subcutaneous tumors, such as angiolipomas, superficial metastatic melanomas, and eccrine spiradenomas [7]. Ultrasonographic findings do not necessarily predict the preoperative diagnosis of SFT. Nevertheless, because the MRI of SFT is relatively nonspecific [5], it is important to accumulate ultrasonographic findings of SFT.

\section{Consent}

The examination of the patient was conducted according to the principles of the Declaration of Helsinki.
The authors certify that they have obtained all appropriate patient consent forms, in which the patients gave their consent for images and other clinical information to be included in the journal. The patients understand that their names and initials will not be published and due effort will be made to conceal their identity, but that anonymity cannot be guaranteed.

\section{REFERENCES}

1. Klemperer P, Rabin CB. Primary neoplasms of the pleura. A report of five cases. Arch Pathol. 1931;11:385-412.

2. Yokoi T, Tsuzuki T, Yatabe Y, Suzuki M, Kurumaya H, Koshikawa T, et al. Solitary fibrous tumour: significance of p53 and CD34 immunoreactivity in its malignant transformation. Histopathology. 1998;32:423-32.

3. Doyle LA, Vivero M, Fletcher CD, Mertens F, Hornick JL. Nuclear expression of STAT6 distinguishes solitary fibrous tumor from histologic mimics. Mod Pathol. 2014;27:390-5.

4. Sekiya M, Yoshimi K, Muraki K, Suzuki K, Dambara T, Uekusa T, et al. Solitary fibrous tumor of the pleura: ultrasonographic imaging findings of 3 cases. Respir Investig. 2013;51:200-4.

5. Tsai SY, Hsu CY, Chou YH, Lai YC, Lin YH, Wang HK, et al. Solitary fibrous tumor of the breast: a case report and review of the literature. J Clin Ultrasound. 2017;45:350-4. 
www.odermatol.com

6. Matsuda T, Haraguchi Y, Shibata-Kikuchi S, Fushimi F, Yamada Y, Oda Y, et al. A case of solitary fibrous tumor. Nishinihon J Dermatol. 2018;80:450-4.

7. Hwang CM, Kang BS, Hong HJ, Lee JY, Suh JH, Han EM, et al. Ultrasonographic features of eccrine spiradenoma. J Ultrasound Med. 2018;37:1267-72.
Copyright by Ai Kajita, et al. This is an open-access article distributed under the terms of the Creative Commons Attribution License, which permits unrestricted use, distribution, and reproduction in any medium, provided the original author and source are credited.

Source of Support: Nil, Conflict of Interest: None declared. 